\title{
A COUPLED NUMERICAL MODEL FOR TSUNAMIS GENERATED BY SUBAERIAL AND SUBMARINE MASS FAILURES
}

\author{
Stéphane Abadie ${ }^{1}$, Stephan Grilli and Stéphane Glockner ${ }^{3}$
}

\begin{abstract}
This paper presents a new numerical model simulating tsunamis generation by landslides. Water, air, and the slide (considered either as a viscous fluid or as a rigid bloc using the penalization method) are described by Navier-Stokes equations, expressed in a unified single fluid approach. The PLIC-VOF method is used to describe the motion of fluid interfaces. We present results for two test cases featuring solid blocks. The first one is that of a semi-elliptical body sliding over a plane slope, without surrounding water. We find, the slide motion is accurately reproduced by the model when the slide viscosity is around $10^{4} \mathrm{~Pa}$.s. For larger viscosity values, the conditioning of the matrix associated with the Navier-Stokes solver is degraded. In the second case, a falling rectangular block generates waves in a flume. We find, wave height and box velocity are in agreement with experiments by Monaghan and Kos (2000). The simulated flow close to the falling box is similar to that observed in experiment, except that the observed main plunging wave and associated air entrainment.do not occur. In future work, the model thus validated will be applied to simulate more realistic cases of tsunamis generated by subaerial landslides.
\end{abstract}

\section{INTRODUCTION}

Tsunamis are typically generated by the co-seismic bottom displacement due to earthquakes. Landslides, however, either submarine or subaerial, can trigger devastative waves as well.

Currently, the accurate computation of tsunami sources appears to be one of the key points in modeling such events. Non linear shallow water wave equations (Jiang and Leblond, 1992) and potential flow calculations based on a Boundary Element Method (Grilli et al., 2002), have been applied with good success to simulate submarine landslide-induced waves.

By contrast, the case of subaerial or partially emerged mass failures is far from being solved, owing to more complex flow features. Indeed, in this case, vertical flow accelerations, dissipation induced by vorticity generation, air entrapment and, above all, the complex coupling between landslide and water flow, all occur and must be properly taken into account in the model.

For this reason, empirical laws based on experiments have usually been used (Walder et al., 2001). Modelling work is also reported for solid block, subaerial

\footnotetext{
1 Laboratoire de Sciences Appliquées au Génie Civil et Côtier, Université de Pau et des Pays de l'Adour, Allée du Parc Montaury, 64600 Anglet, France

2 Department of Ocean Engineering, University of Rhode Island, Narragansett, RI 02882 , USA

3 Laboratoire Trefle - UMR 8508 - Site ENSCPB. 16 Avenue Pey-Berland, 33607 Pessac Cedex, France
} 
landslides in 2D (Heinrich, 1992) and more recently in 3D (Liu et al., 2005). These studies used semi-empirical laws calibrated with experimental data to specify the slide motion.

Here, we present two-dimensional validation cases of direct numerical hydrodynamic simulations, that will eventually allow to simulate the threedimensional flow induced by subaerial landslides. The model uses a three-fluid (air, water, landslide) domain and represents fully viscous effects. Landslides are treated as fluid, whose viscosity law can be adjusted in order to make the third fluid, to the limit, nearly behaving as a solid, or as a fluid with arbitrary, possibly non-Newtonian properties.

\section{THE MODEL}

The hydrodynamic model used here is part of the Computational Fluid Dynamics library Aquilon, developed at the Trefle laboratory. Although the model is three-dimensional, we will only present here two-dimensional validation cases.

\section{The equation system}

The computational domain is made of three fluids : water, air, and a third fluid, which represents the slide. According to the work of Scardovelli and Zaleski (1999), incompressible flows involving several fluids separated by interfaces can be described by a unified "single fluid" formulation :

$$
\begin{gathered}
\nabla \cdot \boldsymbol{U}=0 \\
\rho\left(\frac{\partial \boldsymbol{U}}{\partial t}+(\boldsymbol{U} \cdot \nabla) \boldsymbol{U}\right)=\rho \boldsymbol{g}-\nabla P+\nabla\left(\mu\left(\nabla \boldsymbol{U}+\nabla^{t} \boldsymbol{U}\right)\right)
\end{gathered}
$$

where $\boldsymbol{U}$ is the flow velocity, $\rho$ the density, $\mu$ the dynamic viscosity, $\boldsymbol{g}$ the gravitational acceleration, and $P$ the fluid pressure.

Since there are several fluids in the domain, both density and viscosity are expressed as functions of the location.

The motion of interfaces is represented by advection equations, expressed for a "volume fraction function" quantifying the fraction of each fluid present in interface cells, specifying that these interfaces are moving with the flow. Here, two such equations can be expressed for the two interfaces between the three fluids.

$$
\begin{gathered}
\frac{\partial C_{\text {water }}}{\partial t}+(\boldsymbol{U} \cdot \nabla) C_{\text {water }}=0 \\
\frac{\partial C_{\text {slide }}}{\partial t}+(\boldsymbol{U} \cdot \nabla) C_{\text {slide }}=0
\end{gathered}
$$


where $\mathrm{C}_{\text {water }}\left(\right.$ resp. $\mathrm{C}_{\text {slide }}$ ) is equal to 1 in water (resp. in the slide) and 0 everywhere else.

\section{Numerical procedures}

The coupling between equations (1) and (2) is solved by using the augmented Lagrangian procedure described in Lubin et al. (2006). This method has been adapted from the original method, to better deal with sharp variations in fluid density, due to the presence of several fluids.

The equations obtained are then discretized on a staggered Eulerian grid. A hybrid upwind-centered scheme is used for the advective terms. The implicit time discretization leads to a linear system, which is preconditioned using a Modified and Incomplete LU method, and inverted by applying the iterative BICSTABII algorithm (Van Der Vorst, 1992).

Equations (3) and (4) can be solved by applying a Total Variation Decreasing (TVD) Lax-Wendroff scheme like in Lubin et al. (2006). In the present work, the Peacewise Linear Interface Construction (PLIC) algorithm is employed (Li, 1995). In this method, the interface between water and the two other fluids is reconstructed by using the average value of $\mathrm{C}_{\text {water }}$ in the mesh cells. A piecewise linear approximation of the actual interface is specified. Each segment is then advected across the mesh, using the local fluid velocity. The final stage is to geometrically calculate the new volume fraction of water in each cell. The same procedure is applied to calculate the slide interface volume fraction $\mathrm{C}_{\text {slide }} \cdot \mathrm{C}_{\text {air }}$ is easily deduced from $\mathrm{C}_{\text {water }}$ and $\mathrm{C}_{\text {slide }}$ since the sum of volume fractions in any cell is at most one.

The new volume fractions are finally used to calculate the local density and viscosity in the fluid domain. For cells containing an interface, the following equation are applied :

$$
\begin{aligned}
\rho & =C_{\text {water }} \rho_{\text {water }}+C_{\text {slide }} \rho_{\text {slide }}+C_{\text {air }} \rho_{\text {air }} \\
\mu & =C_{\text {water }} \mu_{\text {water }}+C_{\text {slide }} \mu_{\text {slide }}+C_{\text {air }} \mu_{\text {air }}
\end{aligned}
$$

\section{VALIDATION}

In this paper, we only present two-dimensional validation applications in order to assess the model accuracy. As a proxy for solid subaerial landslides, we focus on waves generated by rigid blocs in idealized geometry, although the model may also easily deal with deformable slides, e.g., represented by Newtonian or non-Newtonian fluids.

The behavior of a fluid as a rigid bloc can theoretically be obtained by 
imposing an infinite value to the viscosity of a Newtonian fluid (Caltagirone and Vincent, 2001). Coupled with a Navier-Stokes solver, this penalization approach allows to treat, in a very natural way, classical problems such as flows around obstacles, or more complex flows generated by moving rigid obstacles. In our case, this penalization method is used with a Navier-Stokes - Volume Of Fluid (VOF) model, to study the coupling between a rigid block sliding or falling into water, and the surrounding fluid and associated free surface.

\section{Simple slide in air}

The first test case consists of a rigid slide moving over an incline. Only two fluids are considered in this simple case : a first fluid representing the rigid slide, for which a very large viscosity is specified and air.

The objectives here are to first assess the influence of the viscosity value on the slide behavior and second to validate the slide kinematics, without any surrounding water effect.

Figure 1 presents a sketch of the case simulated here. The slide is a semiellipse of length $\mathrm{L}=0.395 \mathrm{~m}$, thickness $\mathrm{T}=0.082 \mathrm{~m}$ and gravity center $\mathrm{G}$. The domain is a $4 \mathrm{~m} \times 1.2 \mathrm{~m}$ rectangle and the size of the Cartesian mesh is $300 \times 100$. Free slip conditions are specified on every boundary of the domain. The slope angle $\alpha$ is simply created by specifying an oblique gravity vector with respect to the vertical direction. During computations, after a few constant time steps allowing the slide motion to start, time steps are automatically calculated so that the Courant Friedrich Levy (CFL) number is always lower than 0.5 , thereby ensuring the stability of the PLIC-VOF method.

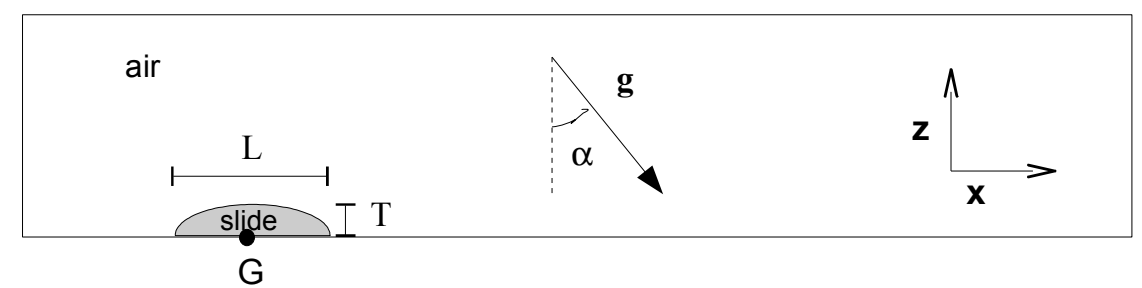

Figure 1 : definition sketch of the first test case of a solid slide on a dry incline.

Since the effect of the surrounding air on the slide dynamics should be very weak, simulation results are compared to the following simple prediction of Newton's law, expressing the free slide of a body over an incline of angle $\alpha$ with the horizontal direction :

$$
x_{G}(t)=\sin (\alpha) g \frac{t^{2}}{2}
$$


Several tests were carried out considering different values of the slide viscosity. These simulations show that too large a value of the viscosity (i.e. greater than $\left.10^{5} \mathrm{~Pa} . \mathrm{s}\right)$, although ensuring the rigidity condition for the slide, leads to a poor agreement with the analytical law. The reason can be found in Caltagirone and Vincent (2001), who showed that large viscosity values degrade the conditioning of the linear system matrix.

Optimal values of the slide viscosity thus appear to be around $10^{4} \mathrm{~Pa}$.s. For lower values, the slide starts deforming during motion. For instance, for $\mu_{\text {slide }}=10^{2}$ Pa.s its longitudinal deformation $\frac{\Delta L}{L}$ reaches about $25 \%$ after the slide has traveled about four times its length. Slide deformations reaches $5 \%$ for $\mu_{\text {slide }}=10^{3}$ Pa.s, and less than $1 \%$ for $\mu_{\text {slide }}=10^{4}$ Pa.s, which is acceptable.

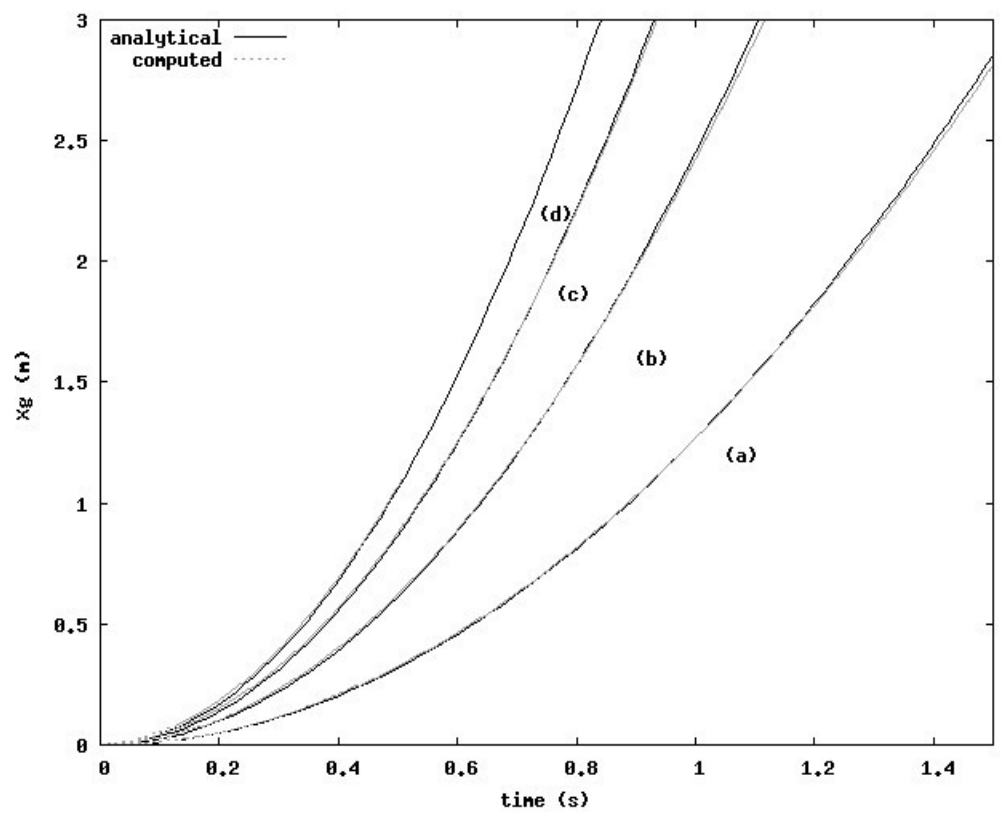

Figure 2 : Slide center of mass motion as a function of time, for different slope values: $\mu_{\text {slide }}=10^{4}$ Pa.s. (a) $\alpha=15^{\circ}$, (b) $\alpha=30^{\circ}$, (c) $\alpha=45^{\circ}$, (d) $\alpha=60^{\circ}$.

Figure 2 presents a comparison of simulation results with the analytical law (7), for $\mu_{\text {slide }}=10^{4}$ Pa.s and considering four different slope values. The 
agreement is excellent with differences nearly indistinguishable in each case, the maximum RMS difference between computations and Eq. (7) being around 0.016 .

\section{Scott Russel's wave generator}

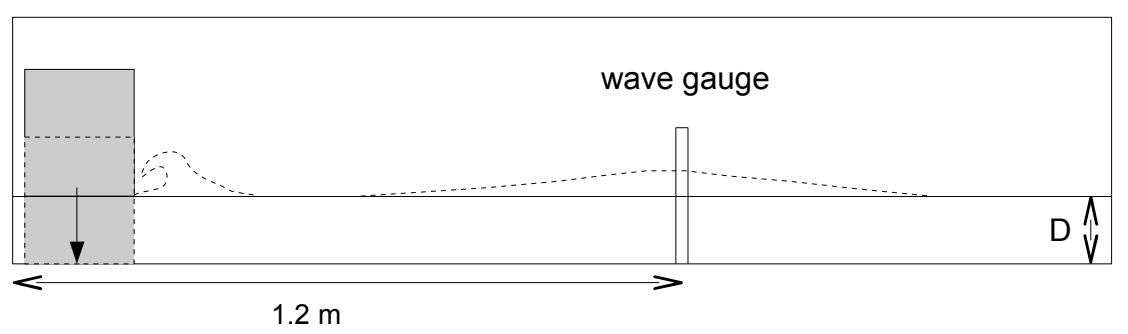

Figure 3 : Sketch of Scott Russel's wave generator problem parameters.

Scott Russel was the first to describe the generation of a solitary wave by a stopping/bouncing barge in a canal, and its constant speed and permanent form during propagation, while following the wave on his horse (Russel, 1844). The configuration of the so-called Scott Russel's wave generator in our model is presented in Figure 3. A rigid rectangular box, initially standing just above the water surface, at the left side of a 2D flume, is released and falls vertically (horizontal motion blocked) into water under gravity effect. This violent motion generates a complex flow pattern close to the box and, eventually, a solitary wave traveling toward the right far from the box not too dissimilar from the flow caused by a rigid subaerial landslide on a steep slope.

This problem was studied experimentally and numerically by Monaghan and Kos (2000). In their paper, the flume length was $9 \mathrm{~m}$, the box of height $=0.4 \mathrm{~m}$, length $0.3 \mathrm{~m}$ and width $0.39 \mathrm{~m}$, weighted $38.2 \mathrm{~kg}$. During the experiment, different flume depths were considered : $\mathrm{D}=0.288 \mathrm{~m}, \mathrm{D}=0.21 \mathrm{~m}$ and $\mathrm{D}=0.116 \mathrm{~m}$.

Monaghan and Kos (2000) provided measurements of the solitary wave height for all depths considered and box velocity and near box flow features, for the $\mathrm{D}=0.21 \mathrm{~m}$ case. They also carried out numerical simulations using a Smooth Particle Hydrodynamics (SPH) model. The following results were found :

- the box velocity increases to about $0.4 \sqrt{(g D)}$, until its lower side reaches half the depth, and then progressively decreases to zero.

- a plunging breaker is created at the right side of the box, degenerating into a first counterclockwise vortex, which travels with the wave down the flume. A second clockwise vortex is also created in the upper part of the plunger, due to the separation of the jet.

- $\quad$ the SPH model was able to reproduce the main flow features. 
The same geometrical dimensions and problem parameters are used for the simulation presented here. The mesh size is $250 \times 130$ but is irregularly spaced. Around the box, the mesh is the finest, with $6 \mathrm{~mm}$ cells. The box viscosity is set to $10^{4}$ Pa.s. Like in the experiment, the box is forced to move vertically by imposing its horizontal velocity to be zero.

Scott Russel's wave generator provides a far more complex test case for the model than the previous one. This time, there is a strong coupling between the box motion and the surrounding water flow. In addition, the water surface deformation highly depends on the correct representation of this coupling.

Figure 4 shows eight snapshots of the water volume fraction and fluid velocity near the moving box. We see, water is pushed mainly from below the box, due to its vertical motion, toward its right side. As the box was initially slightly shifted from the left wall of the numerical flume, some water also moves up at high velocity within the small space between the wall and the box.

While Monaghan and Kos indicated the formation of a plunging breaker, this feature is not very well reproduced in the model, even when using finer meshes. The shape of the upper free surface close to the box agrees well with the reported measurements, but air entrapment does not occur. The reason for this is still not clear and has been investigated as of yet.

The formation of the first counterclockwise vortex is well visible in simulation, at $\mathrm{t}=0.34 \mathrm{~s}$. This vortex follows the wave down the flume with the right timing, as compared to experiments. A second clockwise vortex is, like in the experiment, generated by the backward breaking, which can be observed on the last snapshot.

The model also calculates the air flow, which has quite active features near the top sharp corners of the box. In certain cases, air flow is expected to play a role in wave dissipation processes. Here, strong air vortices are generated at the upper corners of the box and move with it.

The novelty of the present approach for underwater or subaerial landslide tsunami modeling resides in the implicit calculation of the slide/water coupling, using the penalization method. If this coupling worked well (through computed pressure and drag forces), then the slide motion would naturally come out of simulations and it would no-longer be necessary to specify it as a boundary conditions, e.g., based on semi-empirical laws for slide kinematics (Grilli et al., 2002). This would be an improvement since such law needs to be derived based on measurements to be properly calibrated. 

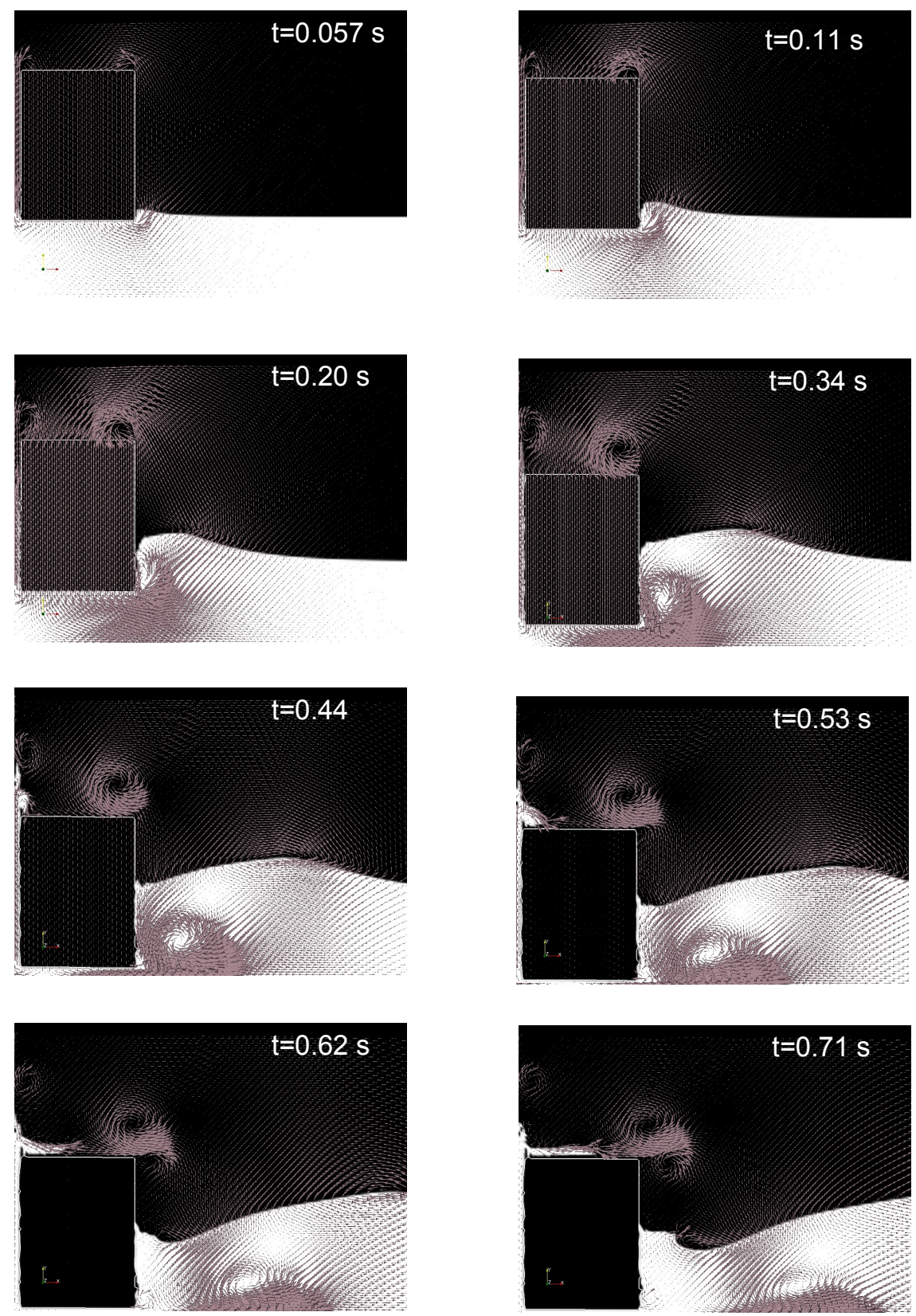

Figure 4 : Simulations of Scott Russel's wave generator $(D=0.21 \mathrm{~m})$. Snapshots of the water volume fraction $\mathrm{C}_{\text {water }}$ (in white) and flow velocity field near the box. 


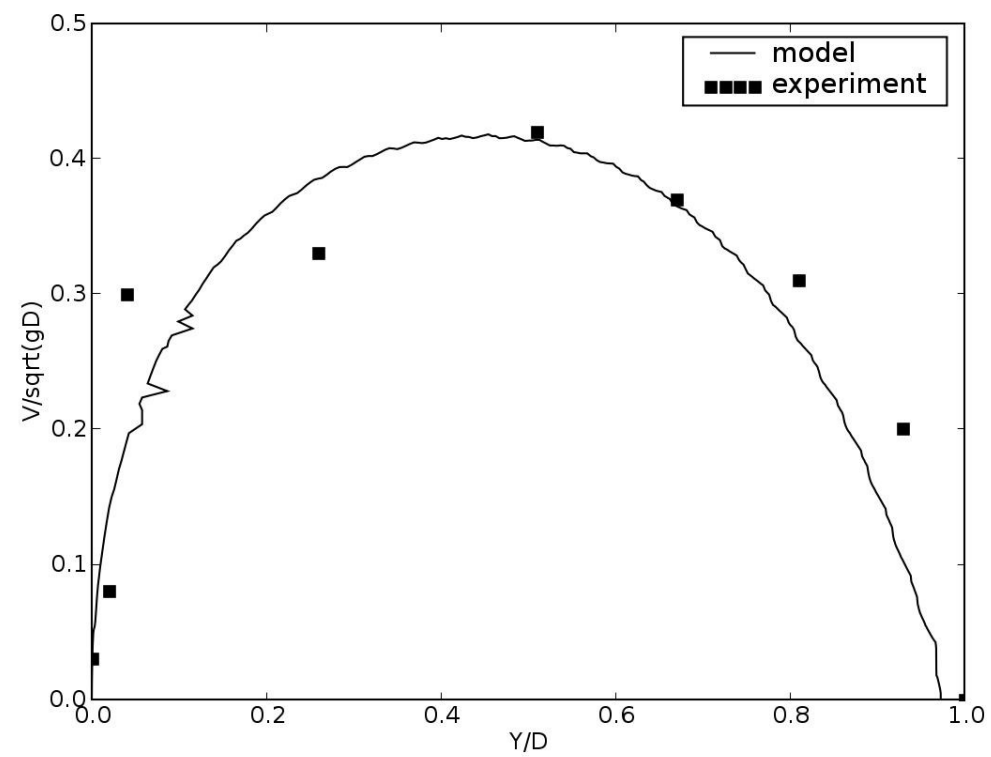

Figure 5 : Non dimensional box velocity as a function of nondimensional depth in model simulation and experiments by Monaghan and $K o s(2000)$ for $D=0.21 \mathrm{~m}$.

Hence, we will now compare the prediction of the box velocity provided in our model to experimental measurements. This is shown in Figure 5 for the case $\mathrm{D}=0.21 \mathrm{~m}$, where we see, the numerical results agree quite well with experiments for the first $70 \%$ of the water column. At the end of the motion, the simulated box motion seems to slow down a little too quickly. At the end of the simulation, a very thin layer of water thus remains stuck under the box, which explains the slight final shift in depth values in the figure.

Another key point of the model is its ability to accurately reproduce the right free surface deformation (i.e. wave shape), by implicitly calculating the slide effect on the flow. Table 1 thus gives the solitary wave height measured in Monaghan and Kos' experiments, with the related accuracy, and the numerical results. Except for the first shallower case $(\mathrm{D}=0.116 \mathrm{~m})$, for which the simulated wave is a bit too small, the two other cases are quite well reproduced by the model, considering the experimental error, even with the relatively coarse mesh we used. Results for the first water depth should likely improve when using a finer model mesh. 
Table 1 : wave heights measured (Monaghan and Kos, 2000) and simulated (present study), 1.2 meters from the left side of the flume for three flume depths.

\begin{tabular}{|c|c|c|c|}
\hline depth $D(m)$ & $\begin{array}{c}\text { wave height } \\
\text { measured }(\mathrm{m})\end{array}$ & $\begin{array}{c}\text { Experimental } \\
\text { Accuracy }(\mathrm{m})\end{array}$ & $\begin{array}{c}\text { wave height } \\
\text { simulated }(\mathrm{m})\end{array}$ \\
\hline 0.116 & 0.109 & \pm 0.02 & 0.0884 \\
\hline 0.21 & 0.092 & \pm 0.01 & 0.097 \\
\hline 0.288 & 0.093 & \pm 0.01 & 0.093 \\
\hline
\end{tabular}

\section{CONCLUSION}

This paper describes a new hydrodynamic model for simulating subaerial landslide tsunamis. The model is based on Navier-Stokes equations, expressed in three fluids (air, water and a very viscous fluid representing the slide), combined with a PLIC-VOF method to express interfacial motion. Equations in water, air, and the landslide are expressed in a unified single fluid formulation. Two test cases were carried out and reported in the paper : the simple case of a rigid bloc sliding over an incline, without surrounding water, and Scott Russel's wave generator case.

The following conclusions can be drawn from this study :

- The slide behaves as a rigid bloc when large values of the viscosity are specified $\left(10^{4}\right.$ Pa.s seems to be an optimal choice since larger viscosity values degrade the matrix associated with the NavierStokes equation solver).

- The simple sliding of a rigid bloc over an incline under the action of gravity is well reproduced by the model.

- In Scott Russel's wave generator case, wave height and box velocity are correctly simulated by the model.

- The main features of the flow close to the falling box are obtained but the main plunging wave and air entrapment are not well reproduced.

Future work will use the present validated model to simulate cases of subaerial and underwater landslide, for which experiments have been performed (Liu et al., 2005; Enet and Grilli, 2003, 2006 ). 


\section{REFERENCES}

Caltagirone, J. P. and Vincent, S., 2001. "Tensorial penalization method for solving Navier Stokes equations". C. R. Acad. Sci. Paris, 329, Série IIb, 607-613.

Enet, F, Grilli, S.T. and Watts, P., 2003. Laboratory Experiments for Tsunamis Generated by Underwater Landslides: Comparison with Numerical Modeling. In Proc. 13th Offshore and Polar Engng. Conf. (ISOPE03, Honolulu, USA, May 2003), 372-379.

Enet, F. and Grilli, S.T. 2006. "Experimental Study of Tsunami Generation by Three-dimensional Rigid Underwater Landslides." J. Waterway Port Coastal and Ocean Engng. (in press).

Grilli, S.T., Vogelmann, S. and Watts, P. 2002. "Development of a 3D Numerical Wave Tank for modeling tsunami generation by underwater landslides". Engng. Analysis Boundary Elemt. 26(4), 301-313.

Heinirich, P., 1992. "Non linear water waves generated by submarine and aerial landslides". J. Waterways, Port Coastal and Ocean Eng. , 118, 249

Jiang, L., Leblond, P. H., 1992. "The coupling of a submarine slide and the surface wave it generates". J. Geophys. Res., 97, 12, pp. 731-744.

Li, J., 1995, Piecewise Linear Interface Calculation). C. R. Acad. Sci,.Paris, série Iib, 320, 391-396.

Liu, P. L.-F., Wu, T.-R., Raichlen, F., Synolakis, C.E. and Borrero, J.C., 2005. "Runup and rundown generated by three-dimensional sliding masses." $J$. Fluid Mech., 536, 107-144.

Lubin, P., Vincent, S., Abadie, S. and Caltagirone, J.P., 2006. " Threedimensional Large Eddy Simulation of air entrainment under plunging breaking waves", Coastal Engineering, Volume 53, issue 8, 631-655.

Monaghan J. J. and Kos A., 2000, "Scott Russell's wave generator", Physics of Fluids, 12, 3, p 622-630.

Russel, J., 1844. "Report on waves", Rep. Brit. Assoc. for the Advancement of Science, 311-390.

Scardovelli, R., Zaleski, S., 1999. "Direct numerical simulation of free surface and interfacial flow", Annu. Rev. Fluid Mech., 31, 567-603.

Van Der Vorst, H. A., 1992. "Bi-csgstab : a fast and smoothly converging variant of bi-cg for the solution of non-symmetric linear systems". SIAM J. Sci. Statist. Comput. 12, 631-644.

Walder, J.S., P. Watts, O.E. Sorensen and K. Janssen, 2001. Tsunami generated by subaerial mass flows. Journal of Geophysical Research, 108, NO. B5, 2236. 


\section{KEYWORDS - ICCE 2006}

A COUPLED NUMERICAL MODEL FOR TSUNAMIS GENERATED BY SUBAERIAL AND SUBMARINE MASS FAILURES

Stéphane Abadie, Stephan Grilli and Stéphane Glockner Abstract 226

solitary wave

tsunami

subaerial

landslide

Scott Russel

Navier-Stokes

VOF

penalization 